\title{
Multicenter Evaluation of Resistance Patterns of Klebsiella pneumoniae, Escherichia coli, Salmonella spp and Shigella spp isolated from Clinical Specimens in Brazil: RESISTNET Surveillance Program
}

Carmen Paz Oplustil, Rogério Nunes, Caio Mendes and the RESISTNET Group
Institutional affiliations: Laboratory of Medical Research - LIM-54- Parasitological and Infectious Diseases Division of the Clinical Hospital of Medical School of University of São Paulo; Pfizer Laboratories, São Paulo, SP, Brazil

\begin{abstract}
Surveillance programs are essential to detect the increase of antimicrobial resistance, and several different programs are being conducted in many countries. The RESISTNET is a surveillance program for bacterial resistance against several antimicrobial agents initiated in 1998 among Latin American countries. In Brazil, several centers were invited to join this surveillance and a total of 11 centers ( 6 from São Paulo and 5 from other states) participated in the study. All results were analyzed using the WHONET program. A total of 894 Escherichia coli, 386 Klebsiella pneumoniae, 70 Shigella spp and 57 Salmonella spp strains were analyzed in this study from April, 1998, to April, 1999. Susceptibility testing was performed by the disk diffusion method using NCCLS 1998 guidelines for several different drugs. For all strains, imipenem was the most effective drug (100\% of the strains were susceptible). Klebsiella pneumoniae presented a high resistance rate to ampicillin $(\mathbf{9 6 . 4 \%})$. The rate of probable ESBL producers among $K$. pneumoniae strains was $36.3 \%$, most of them being isolated from catheters $(58.8 \%)$. Among all Escherichia coli strains analyzed, the highest resistance rate was found for trimethoprim/ sulfamethoxazole (46.9\%) and the majority of the resistant strains were isolated from urine samples (47.8\%). Among Salmonella spp, the resistance rates were low for all antibiotics tested. For Shigella spp strains there was a high resistance to trimethoprim/sulfamethoxazole $(\mathbf{8 0 . 0} \%)$. No resistance to ceftriaxone was observed in these strains. Surveillance of antimicrobial resistance is critical for the successful management of infectious diseases. The results of this survey show significant resistance rates among these bacteria which are responsible for several types of human infections.
\end{abstract}

Key words: Enterobacteria, antimicrobial resistance, surveillance programs, WHONET.

The increase in antimicrobial resistance of bacteria to different antimicrobials leads many countries to initiate surveillance programs [1-3]. From all bacteria that have developed resistance, the Enterobacteriaceae are a group of special concern

Received on 25 August 2000; 14 November 2000.

Address for correspondence: Dr. Carmen Paz Oplustil - Av. General Waldomiro de Lima, 509 Zip Code 04344-070 - São Paulo/SP - Brazil.

The Brazilian Journal of Infectious Diseases 2001;5(1):8-12 (c) 2001 by The Brazilian Journal of Infectious Diseases and Contexto Publishing. All rights reserved. $1413-8670$ due to the high increase of resistance recently observed. Salmonella spp and Shigella spp are clear examples, with an increase in resistance to antibiotics that are considered drugs of choice in the treatment of infections caused by these bacteria. Some strains of Klebsiella pneumoniae and Escherichia coli produce different enzymes (ESBL, carbapenases, beta-lactamases) that are changing the therapeutic usefulness of some antimicrobials.

There are some situations that physicians and microbiologists must know in order to have a better understanding of the dissemination and control of bacterial resistance. Surveillance programs are essential 
to meet this goal. In 1998, the RESISTNET program was initiated to analyze the resistance patterns of several bacteria from Latin American countries.

This study presents data pertaining to antimicrobial resistance of Escherichia coli, Klebsiella pneumoniae, Shigella spp, and Salmonella spp collected from 11 centers around Brazil. All data was entered into the WHONET program [4]. The WHONET program is a computer program designed to monitor and manage information about bacterial resistance by merging from many centers and providing an overview of global antimicrobial resistance patterns.

\section{Materials and Methods}

In this study, resistance data were collected from April, 1998, to April, 1999, from 11 centers in Brazil. Six centers were located in the city of São Paulo (Santa Casa de Misericórdia de São Paulo, Hospital São Paulo, Laboratório de Investigação Médica - LIM54- Divisão de Moléstias Infecciosas e Parasitárias do HC FMUSP, Hospital Emilio Ribas, Faculdade de Ciências Farmacêuticas USP and Hospital Heliópolis). The other five centers were from different cities around the country (Brasilia - Laboratório Exame; Curitiba Hospital das Clínicas de Curitiba; Salvador - Hospital Aliança; Fortaleza - Laboratório Louis Pasteur; Porto Alegre - Hospital das Clínicas de Porto Alegre).

A total of 894 Escherichia coli, 386 Klebsiella pneumoniae, 70 Shigella spp, and 57 Salmonella spp strains from inpatients and outpatients from varied anatomical sites were analyzed in this study. Susceptibility tests were performed by the disk diffusion method and the results were evaluated according to the NCCLS 1998 guidelines [5]. The following drugs were tested against E.coli and K. pneumoniae: ampicillin, cephalotin, ceftriaxone, ciprofloxacin, gentamicin, imipenem, ampicillin/sulbactam, and trimethoprim/sulfamethoxazole. For Salmonella spp and Shigella spp, the following drugs were tested: ampicillin, chloramphenicol, ceftriaxone, ciprofloxacin and trimethoprim/sulfamethozaxole. All results were entered into the WHONET program (WHONET4).
Quality control was performed with the following ATCC strains: Staphylococcus aureus 25923, E. coli 25922 , and $P$. aeruginosa 27853.

\section{Results and Discussion}

The resistance rates for all 4 bacteria analyzed in this study are presented in Figure 1. Table 1 shows the difference in the resistance rates for these bacteria isolated from outpatients and inpatients.

Antimicrobial Susceptibility of Klebsiella pneumoniae

From the 386 strains of $K$. pneumoniae analyzed, 270 (70\%) were from inpatients and $116(30 \%)$ were from outpatients. Among all K. pneumoniae analyzed, $31.9 \%$ (123) were isolated from urine, $13.7 \%$ (53) from blood, $9.3 \%$ (36) were isolated from samples of the lower respiratory tract, 5.7\% (22) from abscesses, $4.1 \%$ (17) from catheters and $35.4 \%$ were isolated from several other clinical specimens.

Imipenem was the most efficient drug against $K$. pneumoniae (all strains were susceptible). The percentage of resistance to ciprofloxacin was $2.2 \%$ and resistance to trimethoprim/sulfamethoxazole(TSX) was $33.3 \%$. The percentage of resistance among outpatients to ampicillin/sulbactam was $11.3 \%$ and, to ampicillin alone, the resistance was $94.5 \%$. A similar profile was observed for inpatients, where we had $33.7 \%$ resistance to ampicillin/sulbactam, and $97 \%$ to ampicillin alone. There was $3.9 \%$ resistance to cefoxitin, probably the same percentage of ampC K. pneumoniae strains. The highest percentage of $K$. pneumoniae strains producing ampC $(5.9 \%)$ were found in strains isolated from catheters. Among all strains tested, we found $36.3 \%$ of strains to be possible ESBL producers when NCCLS criteria (inhibition zones $\leq 25 \mathrm{~mm}$ ) were used to evaluate ceftriaxone activity. From the 53 strains of K. pneumoniae isolated from blood samples, $47.2 \%$ were probable ESBL producers and over half (58.8\%) of those isolated from catheters were also probable ESBL producers. In all strains isolated from urine samples, $24.4 \%$ were probable ESBL producers. From 
the 123 strains isolated from urine samples, 41 (33\%) were from outpatients and $17.1 \%$ of these strains were found to be probable ESBL producers. These data indicate that this is an important point to be considered when treating this kind of infection. The high rates of ESBL producing strains in Latin American countries have been described in several other studies $[2,6,7]$. The rates found in these surveillance studies show similar percentages among $K$. pneumoniae strains. In a multicenter study from 5 European countries, the percentage of probable ESBL producers was lower than the percentage found in our study. In that study the highest percentages were found in Portugal (36.0\%) and in France (26.0\%) [1]. Another interesting observation in this study was the important difference in the resistance rates for aminoglycosides between strains isolated from inpatients and outpatients (Table 1). This could be due to the extensive use of this class of antimicrobials in the hospital environment.

\section{Antimicrobial Susceptibility of Escherichia coli}

From the 894 strains of E. coli analyzed $67.1 \%$ (600) were isolated from inpatients and 32.9\% (294) from outpatients (Table 1). Of all the strains of E. coli analyzed, $52.7 \%$ (471) were isolated from urine samples, 7.6\% (68) from abscess, 6.4\% (57) from blood, $2.8 \%$ (25) from surgical wounds, $0.2 \%$ (2) from catheters, and $30.3 \%$ (271) were isolated from several other clinical specimens. For all strains, there was a $55.0 \%$ resistance rate to ampicillin; however, the association with sulbactam showed a significantly lower resistance rate $(12.0 \%)$.

A similar profile was observed when we analyzed strains from inpatients and outpatients. Among inpatients, all strains showed $13.7 \%$ resistance to ampicillin/sulbactam and $55.9 \%$ resistance to ampicillin alone. There was a $46.9 \%$ resistance to TSX among all strains (Figure 1). A higher percentage of strains resistant to TSX was observed in strains isolated from urine samples (47.8\%). Cephalotin is another drug commonly used for the treatment of urinary infections in Brazil, and we observed $11.5 \%$ resistance and $32.5 \%$ intermediate resistance to this drug. This may be due to the extensive use of cephalotin for treating this type of infection. The scenario was similar for strains isolated from blood cultures, with all strains presenting a high percentage of resistance to cephalotin (23.6\%) and to TSX (41.8\%).

For all of the Ecoli strains analyzed, there was a $5.9 \%$ resistance rate to ciprofloxacin. In strains isolated from urine, the percentage increased to $6.4 \%$ and, for strains isolated from blood cultures, the resistance rate was $5.5 \%$. Based on the same criteria used to analyze $K$. pneumoniae ESBL producer strains, there was a lower percentage of $E$. coli strains probable ESBL producers (8.0\%). This percentage is lower than the one presented by Gales, et al. [6] but very similar to the rate found by Hsiung, et al. [7]. There was no important difference between the resistance rates of $E$. coli strains isolated from inpatients and outpatients (Table 1).

Antimicrobial Susceptibility of Salmonella spp and Shigella spp

Of all Salmonella spp strains analyzed, 66.7\% (38) were from inpatients and 33.3\% (19) from outpatients. For Shigella spp strains, $81.4 \%$ (57) were from inpatients and $18.6 \%$ (13) from outpatients. Of the 57 strains of Salmonella spp analyzed, $35.0 \%$ were isolated from blood, $49.1 \%$ from stool, and $15.9 \%$ from other sites (urine, abscess, bile). Of the 70 strains of Shigella spp, $20.0 \%$ were isolated from blood, $74.3 \%$ from stool, and $5.7 \%$ from other sites. Shigella sonnei was the most frequent species isolated (63.0\%). All Salmonella species were susceptible to TSX, ceftriaxone and ciprofloxacin (Figure1) (Table 1). The rate of resistance to ampicillin $(7.0 \%)$ is rather low when compared to the resistance rates found in other Latin American countries (Argentina 60.3\%, Colombia $20.0 \%$, Mexico 16.7\%) [8]. If we analyze the resistance patterns of the isolates obtained from blood and stool to ampicillin, we note that there is no resistance among strains isolated from blood but among strains isolated from stool, $10.7 \%$ are resistant. This might be because some of these infections are treated with ampicillin and other common drugs that are very popular in 
Figure 1. Antimicrobial resistance rates among all strains analyzed (1,407 strains)

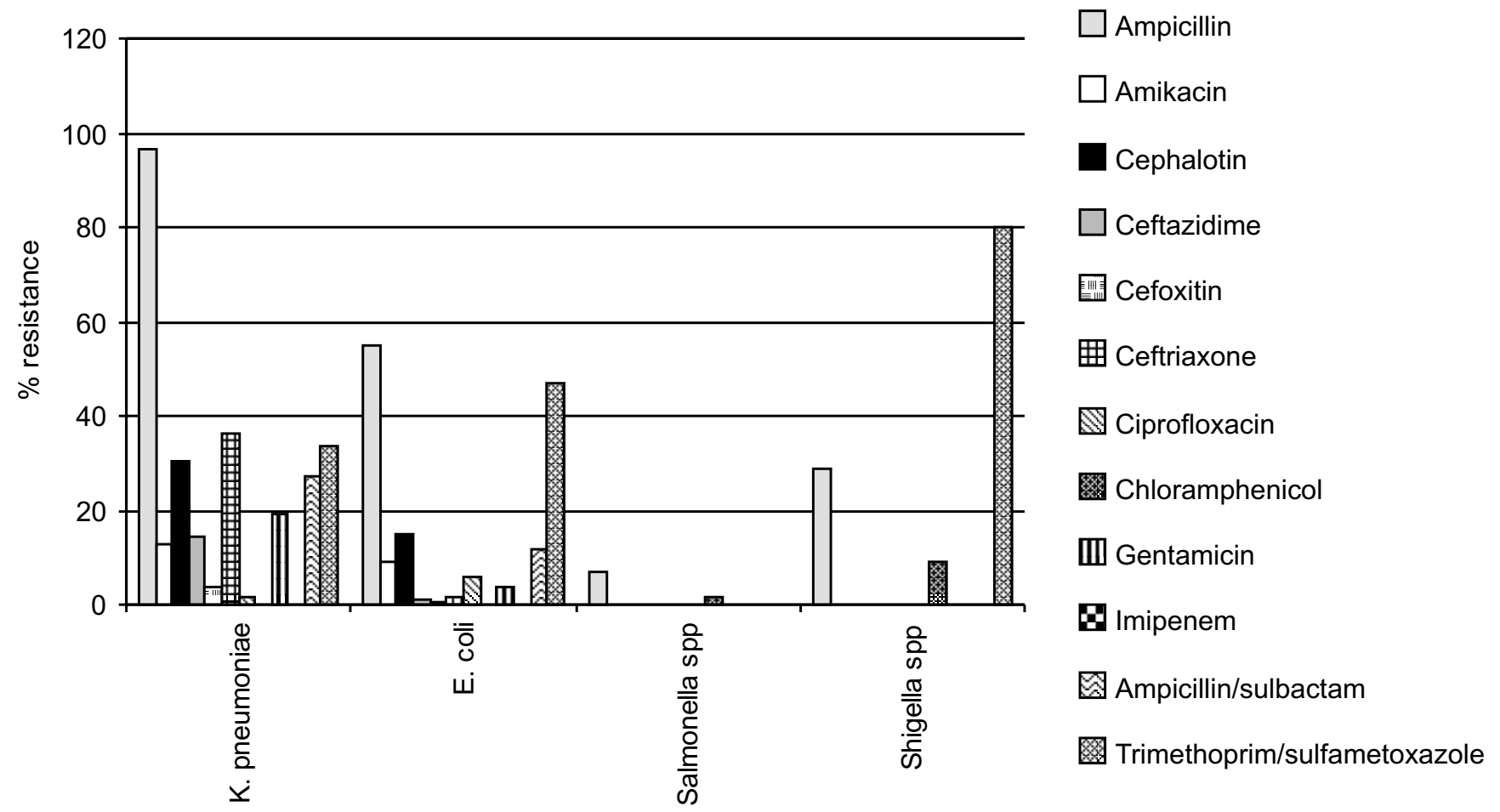

Table 1: Percentage of resistance of K. pneumoniae, E.coli, Salmonella spp and Shigella spp from inpatients and outpatients

\begin{tabular}{|c|c|c|c|c|c|c|c|c|}
\hline \multirow[t]{2}{*}{ Antimicrobial } & \multicolumn{2}{|c|}{ K. pneumoniae } & \multicolumn{2}{|c|}{ E. coli } & \multicolumn{2}{|c|}{ Salmonella spp } & \multicolumn{2}{|c|}{ Shigella spp } \\
\hline & $\begin{array}{c}\text { In } \\
(n=270)\end{array}$ & $\begin{array}{c}\text { Out } \\
(n=116)\end{array}$ & $\begin{array}{c}\text { In } \\
(n=600)\end{array}$ & $\begin{array}{c}\text { Out } \\
(n=294)\end{array}$ & $\begin{array}{c}\text { In } \\
(n=38)\end{array}$ & $\begin{array}{c}\text { Out } \\
(n=19)\end{array}$ & $\underset{(n=57)}{\text { In }}$ & $\begin{array}{c}\text { Out } \\
(n=13)\end{array}$ \\
\hline Ampicillin & $97.0 * *$ & 94.5 & 55.9 & 53.1 & 5.3 & 10.5 & 33.3 & 7.7 \\
\hline Amikacin & 16.3 & 4.3 & 1.2 & 0.3 & - & - & - & - \\
\hline Cephalotin & 38.3 & 12.9 & 15.4 & 13.7 & - & - & - & - \\
\hline Ceftazidime & 18.5 & 4.3 & 0.8 & 1.4 & - & - & - & - \\
\hline Cefoxitin & 4.8 & 1.7 & 0.3 & 0.7 & - & - & - & - \\
\hline Ceftriaxone* & 42.2 & 22.4 & 9.0 & 5.8 & 0 & 0 & 0 & 0 \\
\hline Chloramphenicol & - & - & - & - & 0 & 5.6 & 10.9 & 0 \\
\hline Ciprofloxacin & 2.2 & 0 & 5.0 & 7.9 & 0 & 0 & 0 & 0 \\
\hline Gentamicin & 25.2 & 5.2 & 4.4 & 3.1 & - & - & - & - \\
\hline Imipenem & 0 & 0 & 0 & 0 & - & - & - & - \\
\hline $\begin{array}{l}\text { Ampicillin/ } \\
\text { Sulbactam }\end{array}$ & 33.7 & 11.3 & 13.7 & 8.6 & - & - & - & - \\
\hline $\begin{array}{l}\text { Trimethoprim } \\
\text { Sulfamethozaxole }\end{array}$ & 39.3 & 20.7 & 47.4 & 45.7 & 0 & 0 & 82.5 & 69.2 \\
\hline
\end{tabular}

In: inpatients; Out: outpatients;-: not tested.*: considering ceftriaxone $\leq 25 \mathrm{~mm}$; **: percentage of resistance. 
some countries. Chloramphenicol is another antimicrobial that presented different resistance rates in blood and stool isolates, with $0 \%$ and $4 \%$ resistance, respectively.

There was a high resistance rate to TSX among Shigella species $(80.0 \%)$, with similar resistance in strains isolated from blood (76.9\%) and stool (83.0\%). All strains showed $28.6 \%$ resistance to ampicillin. Shigella spp isolated from stool samples showed $30.2 \%$ resistance to ampicillin, and those isolated from blood showed $23.1 \%$ resistance. The resistance rates to ampicillin are higher in other Latin American countries than the ones we found in this study [8]. The same was observed with chloramphenicol where we observed a $9.0 \%$ resistance, while the resistance rates were much higher in other countries (over 40.0\%). No resistance was observed to ceftriaxone.

The results of this study show the importance of these 4 bacteria as causes of infections. Furthermore, we demonstrated that important antimicrobial resistance rates exist among the 4 isolates analyzed. In making therapeutic choices, it is very important to know the antimicrobial resistance rates of these bacteria when selecting empiric therapy for a patient. Surveillance studies play a very important role for the management of infectious diseases. It is important to conduct these studies to obtain data which allow us to recognize the susceptibility patterns of different bacteria species.

\section{Acknowledgements}

The RESISTNET-Group: H. S. Sader (São Paulo Hospital); C. Mendes (LIM-54, Infectious Diseases Department, HC-FMUSP, São Paulo); I. Mimiça (Santa Casa da Misericórdia de São Paulo); Flávia Rossi (Emilio Ribas Hospital, São Paulo); E. Mamizuca (Faculty of Science Pharmaceuthical, USP, São Paulo); A. Segura (Exame Laboratory, Brasília); L. Della Costa (Clinical Hospital, Curitiba); José Francisco (Aliança Hospital, Salvador); T. Bandeira (Louis Pasteur Laboratory, Fortaleza); A. Barth (Clinical Hospital, Porto Alegre).

\section{References}

1. Hanberger H., Garcia-Rodriguez J.A., Gobernado M., et al. Antibiotic susceptibility among aerobic Gram-negative bacilli in intensive care units in 5 European countries. JAMA 1999;281:67-71.

2. Gales A.C., Jones R.N., Pfaller M.A, et al. Two year assessment of the pathogens frequency and antimicrobial resistance patterns among organisms isolated from skin and soft tissue infections in Latin American Hospitals. Results from the SENTRY Antimicrobial Surveillance Program. Int J Infec Dis 2000; 4 :75-84.

3. Bantar C., Famiglietti A., Goldberg M., the Antimicrobial Committee and the National Surveillance Program (SIR) Participants Group. Three-Year surveillance study of nosocomial bacterial resistance in Argentina. Int J Infect Dis 2000; $4: 85-90$.

4. Stelling J.M., O'Brien T. Surveillance of Antimicrobial Resistance: The WHONET program. Clin Infect Dis. 1997; Supple 1: S157-68.

5. National Committee for Clinical Laboratory Standards (NCCLS). Performance Standards for Antimicrobial Susceptibility Testing; Eighth Informational Supplement M100-S8, Wayne, PA: NCCLS, 1998.

6. Gales A.C, Bolmstron A., Jones R.N., et al. Prevalence, antimicrobial susceptibility, and molecular typing of extended-spectrum beta-lactamases producing Klebsiella pneumoniae from Brazil. In Program and abstracts: 37th Interscience Conference on Antimicrobial Agents and Chemotherapy. Toronto, Canada, 1997.

7. Hsiung Y.J., Sinto S.I., Oplustil C.P., Mendes C.M.F. Evaluation of combination of cefpodoxime and double disk method as initial screening test for detection of ESBL in Escherichia coli and Klebsiella pneumoniae. In Program abstract: 99th American Society for Microbiology Meeting: Chicago, 1999.

8. The RESISTNET Collaborative Group. Comparative evaluation of the susceptibility to antimicrobials of Escherichia coli, Klebsiella pneumoniae, Salmonella spp and Shigella spp isolated from clinical specimens in Latin America. In Program and Abstracts: IDSA, Philadelphia, USA, 1999. 